\title{
LMI-Based Output Feedback Control Design in the Presence of Sporadic Measurements
}

\author{
Roberto Merco, Francesco Ferrante, Ricardo G. Sanfelice, and Pierluigi Pisu
}

\begin{abstract}
This paper considers the problem of stabilizing a linear time-invariant system in the presence of plant measurements that are available in an intermittent fashion. We propose a dynamic output feedback controller equipped with a holding device that is a linear time-invariant system whose state is reset when a new measure is available. We provide an LMI-based design procedure for the co-design of the dynamic controller and holding device parameters. Our approach relies on Lyapunov theory for hybrid systems and addresses the stability analysis in a way that is reminiscent of an "inputto-state stability small gain" philosophy. The effectiveness of the proposed LMI-based design is showcased in a numerical example.
\end{abstract}

\section{INTRODUCTION}

Over the past few decades, Networked Control Systems (NCSs) has been a very active research area; see, e.g., [1]. A peculiarity of NCSs is the capability of sharing information, such as plant measurements and control signals, through a network. Due to the packet-based nature of the network, information between the plant and the controller is exchanged only at discrete transmission events. In particular, due to digital devices and the limited bandwidth and unreliability of the network, new data is generally available in an aperiodically sampled fashion.

Aperiodic sampled-data systems have been studied in several areas of control theory; see, e.g., [2] for a comprehensive survey. The main approaches developed to guarantee stability of sampled-data control systems are the input-delay approach, the lifting approach, and the impulsive system approach. The input-delay approach models the NCS as a continuous-time system subject to a time-varying input delay. In the lifting approach, the sampled-data control problem is converted into an equivalent finite-dimensional discrete-time

R. Merco and P. Pisu are with the Automotive Department of Clemson University, Greenville SC 29607 USA. Email: rmerco@clemson.edu, pisup@clemson.edu.

F. Ferrante is with Univ. Grenoble Alpes, CNRS, GIPSA-lab, F-38000 Grenoble, France. Email: francesco.ferrante@gipsa-lab.fr.

R. G. Sanfelice is with Electrical and Computer Engineering Department, University of California, Santa Cruz, CA 95064, USA. Email: ricardo@ucsc.edu.

Research by R. Merco and P. Pisu is supported by the National Science Foundation (NSF) under Grant No. CNS-1544910. Any opinions, findings and conclusions or recommendations expressed in this material are those of the authors and do not necessarily reflect the views of the National Science Foundation. Research by F. Ferrante is funded in part by ANR via project HANDY, number ANR-18-CE40-0010. Research by R. G. Sanfelice has been partially supported by the NSF under Grant no. ECS-1710621 and Grant no. CNS-1544396, by the Air Force Office of Scientific Research under Grant no. FA9550-16-1-0015, Grant no. FA9550-19-1-0053, and Grant no. FA9550-19-1-0169, and by CITRIS and the Banatao Institute at the University of California. control problem. The third approach consists in modeling sampled-data systems as impulsive systems.

Another possible design approach consists of considering the controller as a more general dynamical system interconnected to the plant, whose input is the sampled plant measurement and output is the control action to send to the plant. An LMI-based design of a discrete-time linear timeinvariant dynamic output feedback controller is proposed in [3]. Donkers et al. [4], instead, propose an LMI-based synthesis that results in a switched controller. Fridman et al. [5] present a controller design method relying on a timedelay approach.

In our previous work [6], we propose an observer-based control scheme where both measurements and control input are affected by intermittent behavior and zero-order hold (ZOH) devices are employed. Such a holding device is employed to convert impulsive signals, such as measurements and control actions, to continuous-time signals. As shown in [7] and references therein, many other holding functions can be employed. The benefits of considering more general holding functions are improved robustness as well as enlarged maximal admissible transmission intervals. To this end, in this paper, we consider general holding devices included in NCSs as in Fig. 1 where only the sensing path of the closed-loop system is subject to network communication, and we propose a methodology for the co-design of output feedback dynamic controller and holding device. Notice that the control systems architecture considered in this paper, known as one-channel feedback NCS [1], constitutes a relevant case study since it can capture several configurations of NCSs [1, Section III.A]. More precisely, we propose a hybrid control scheme constituted by the cascade of a "generalized" holding device and a dynamic controller. The proposed general holding device, which is not a $\mathrm{ZOH}$, is a linear time-invariant system whose state is reset to the plant measurement whenever a new transmission occurs. This general holding device generates a continuous-time signal that feeds a linear time-invariant dynamic controller. Our approach leads to a computationally efficient co-design of controller and holding device parameters via LMIs.

One of the main novelties of our methodology is that we consider the closed-loop NCS as a feedback interconnection of two dynamical systems: the "networked-free" closedloop continuous-time system and the network-induced error impulsive system. Seeing the NCS closed-loop from this perspective allows us to address stability analysis via an approach that is reminiscent of an "input-to-state stability small gain" philosophy. A similar approach is employed in 
[8] for the analysis of the stability of NCS. As a difference from [8], stability conditions obtained in this paper enable controller design via the solution to matrix inequalities. In particular, following the general approach in [9] and [10], we formulate a control design algorithm based on the solutions to LMIs coupled with a parameter line search. These results are preliminary work towards a more comprehensive methodology capable of including, for example, $\mathcal{H}_{\infty}$ performance.

The remainder of the paper is organized as follows. Section II introduces the control problem and presents the modeling of the NCS. Stability conditions and controller design are presented in Section III. Section IV shows the effectiveness of the approach in a numerical example. Due to space limitations, proofs of the results will be published elsewhere.

\section{A. Notation}

The set $\mathfrak{N}_{>0}$ is the set of strictly positive integers, $\mathfrak{N}=$ $\mathfrak{N}_{>0} \cup\{0\}, \mathfrak{R}$ is the set of real numbers, $\mathfrak{R}_{\geq 0}$ is the set of nonnegative real numbers, $\mathbb{C}$ is the set of complex numbers. Given $z \in \mathbb{C}, \mathfrak{R e}(z)$ denotes the real part of $z$. The Euclidean space of dimension $n$ is represented by $\Re^{n}, \mathfrak{R}^{n \times m}$ is the set of $n \times m$ real matrices. Give any $A \in \mathfrak{R}^{n \times m}, A^{\top}$ denotes the transpose of $A, A^{-\top}=\left(A^{\top}\right)^{-1}$ (when $A$ is nonsingular), $\operatorname{He}(A)=A+A^{\top}$, and $\operatorname{spec}(A)$ denotes the spectrum of $A$. The identity matrix is denoted by $\mathbf{I}$. The symbol $\mathrm{S}_{+}^{n}$ represents the set of $n \times n$ symmetric positive definite matrices. For a symmetric matrix $A, A \succ 0$ and $A \succeq 0(A \prec 0$ and $A \preceq 0)$ means that $A(-A)$ is, respectively, positive definite and positive semidefinite, and $\lambda_{\min }(A)$ and $\lambda_{\max }(A)$ denote, respectively, the smallest and the largest eigenvalue. In partitioned symmetric matrices, the symbol $\bullet$ represents a symmetric block. For a vector $x \in \mathfrak{R}^{n}$, $|x|$ denotes the Euclidean norm. Given two vectors $x$ and $y$, we use the equivalent notation $(x, y)=\left[x^{\top}, y^{\top}\right]^{\top}$. Given a vector $x \in \mathfrak{R}^{n}$ and a nonempty set $\mathcal{A}$, the distance of $x$ to $\mathcal{A}$ is defined as $|x|_{\mathcal{A}}=\inf _{y \in \mathcal{A}}|x-y|$. For any function $z: \mathfrak{R} \rightarrow \mathfrak{R}^{n}$, we denote $z\left(t^{+}\right):=\lim _{s \rightarrow t^{+}} z(s)$ when it exists.

\section{Problem Statement and Solution Outline}

\section{A. System Description}

We consider a plant $\mathcal{P}$ described by continuous-time linear time-invariant dynamics of the form

$$
\mathcal{P}: \quad \dot{x}_{p}=A_{p} x_{p}+B_{p} u, \quad y=C_{p} x_{p}
$$

where $x \in \mathfrak{R}^{n_{p}}$ represents the state of the plant, $u \in \mathfrak{R}^{n_{u}}$ the control input, and $y \in \mathfrak{R}^{n_{y}}$ is the output of the plant. Matrices $A_{p}, B_{p}$, and $C_{p}$ are given and of appropriate dimensions. We study a setup, depicted in Fig. 10 in which $u$ is a continuous-time signal, whereas $y$ is measurable only at some time instances $t_{k}, k \in \mathfrak{N}_{>0}$, not known in advance. In particular, we assume that the sequence $\left\{t_{k}\right\}_{k=1}^{\infty}$ is strictly increasing and unbounded, and that there exist two positive real scalars $T_{1} \leq T_{2}$ such that

$$
0 \leq t_{1} \leq T_{2}, \quad T_{1} \leq t_{k+1}-t_{k} \leq T_{2} \quad \forall k \in \mathfrak{N}_{>0}
$$

The lower bound on $T_{1}$ in condition (2) introduces a strictly positive minimum time in between consecutive measurements. As such, this avoids the existence of Zeno behaviors, which are unwanted in practice. Moreover, $T_{2}$ defines the Maximum Allowable Transfer Interval (MATI).

Given the plant $\mathcal{P}$ and the measurement setup above, the problem we solve in this paper is the design of an output feedback dynamic controller such that the closed-loop NCS has an appropriately defined set exponentially stable with the largest achievable value of $T_{2}$. This set necessarily enforces the state $x_{p}$ of $\mathcal{P}$ to be zero.

\section{B. Proposed Controller}

Since the presence of the network results into an intermittent stream of plant measurements, we propose a control scheme, depicted in Fig. 1, constituted by a dynamic controller $\mathcal{K}$ and a holding device $\mathcal{J}$. In particular, in the proposed control system, the plant $\mathcal{P}$ is stabilized by a dynamic controller $\mathcal{K}$ that relies on the continuous-time signal $\hat{y}$ generated by the holding device $\mathcal{J}$.

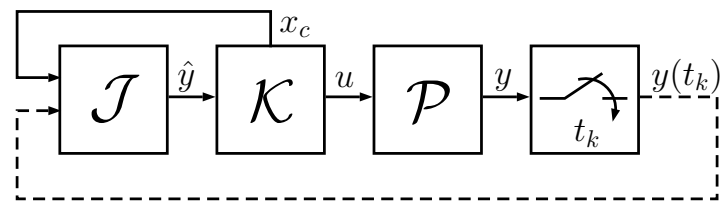

Fig. 1: Schematic representation of the NCS considered in this paper. Solid lines represent continuous-time signals, whereas the dashed line depicts sporadic measurements.

The continuous-time dynamic controller $\mathcal{K}$ we design is given by:

$$
\mathcal{K}\left\{\begin{array}{l}
\dot{x}_{c}=A_{c} x_{c}+B_{c} \hat{y} \\
u=C_{c} x_{c}+D_{c} \hat{y}
\end{array}\right.
$$

where $x_{c} \in \mathfrak{R}^{n_{x_{c}}}$ is the controller state and $\hat{y} \in \mathfrak{R}^{n_{y}}$ is the state of holding device $\mathcal{J}$. By making use of the last received measurement of the plant output and of the controller state (whose value is available at all time), the holding device $\mathcal{J}$ generates an intersample signal that is use to feed the controller $\mathcal{K}$. In particular, $\mathcal{J}$ is described by the following dynamics for all $k \in \mathfrak{N}_{0}$ :

$$
\mathcal{J} \begin{cases}\dot{\hat{y}}(t)=H \hat{y}(t)+E x_{c}(t)+C_{p} B_{p} u(t) & \forall t \neq t_{k} \\ \hat{y}\left(t^{+}\right)=y(t) & \forall t=t_{k}\end{cases}
$$

The operating principle of the holding device $\mathcal{J}$ is as follows. The arrival of new measurements instantaneously updates $\hat{y}$ to $y$. In between updates, $\hat{y}$ evolves according to the continuous-time dynamics in (4) and its value is continuously used by controller $\mathcal{K}$. Matrices $A_{c}, B_{c}, C_{c}, D_{c}, H$, and $E$ are controller parameters that need to be designed. Notice that the traditional $\mathrm{ZOH}$ device constitutes a particular case of the holding device introduced in (4). Indeed, the model in (4) represents a class of generalized holding devices. 


\section{Hybrid Modeling}

The closed-loop system in Fig. 1 can be modeled as an impulsive system with jumps in $\hat{y}$. In particular, for all $k \in$ $\mathfrak{N}_{0}$ one obtains

$$
\begin{aligned}
& \left.\begin{array}{l}
\dot{x}_{p}(t)=f_{p}\left(x_{p}(t), x_{c}(t), \hat{y}(t)\right) \\
\dot{x}_{c}(t)=f_{c}\left(x_{c}(t), \hat{y}(t)\right) \\
\dot{\hat{y}}(t)=f_{y}\left(x_{c}(t), \hat{y}(t)\right)
\end{array}\right\} \forall t \neq t_{k} \\
& x_{p}\left(t^{+}\right)=x_{p}(t) \\
& x_{c}\left(t^{+}\right)=x_{c}(t) \\
& \hat{y}\left(t^{+}\right)=C_{p} x_{p}(t)
\end{aligned}
$$

where $f_{p}\left(x_{p}, x_{c}, \hat{y}\right):=A_{p} x_{p}+B_{p} C_{c} x_{c}+B_{p} D_{c} \hat{y}, f_{c}\left(x_{c}, \hat{y}\right):=$ $A_{c} x_{c}+B_{c} \hat{y}$, and $f_{y}\left(x_{c}, \hat{y}\right):=\left(H+C_{p} B_{p} D_{c}\right) \hat{y}+(E+$ $\left.C_{p} B_{p} C_{c}\right) x_{c}$. To devise a design algorithm for $\mathcal{K}$ and $\mathcal{J}$, we model the impulsive system in (5) within the hybrid system framework in [11]. To this end, we augment the state of the closed-loop system with the auxiliary variable $\tau \in \mathfrak{R}_{\geq 0}$, which is a timer that keeps track of the duration of intervals in between transmissions of new measurement data. As in [10], to enforce (2), we make $\tau$ decrease as ordinary time $t$ increases and, whenever $\tau=0$, reset it to any point in $\left[T_{1}, T_{2}\right]$. The whole closed-loop system composed by the states $x_{p}, x_{c}, \hat{y}$, and $\tau$ can be represented by the following hybrid system:

$$
\begin{cases}\dot{\xi}=f_{\xi}(\xi) & \xi \in \mathcal{C} \\ \xi^{+} \in G_{\xi}(\xi) & \xi \in \mathcal{D}\end{cases}
$$

where $\xi:=\left(x_{p}, x_{c}, \hat{y}, \tau\right) \in \mathfrak{R}^{n_{\xi}}$, with $n_{\xi}:=n_{x_{p}}+n_{x_{c}}+$ $n_{y}+1$, is the state of the hybrid system,

$$
\begin{gathered}
f_{\xi}(\xi):=\left(f_{p}\left(x_{p}, x_{c}, \hat{y}\right), f_{c}\left(x_{c}, \hat{y}\right), f_{y}\left(x_{c}, \hat{y}\right),-1\right) \quad \forall \xi \in \mathcal{C} \\
G_{\xi}(\xi):=\left(x_{p}, x_{c}, C_{p} x_{p},\left[T_{1}, T_{2}\right]\right) \quad \forall \xi \in \mathcal{D}
\end{gathered}
$$

and where the flow set $\mathcal{C}$ and the jump set $\mathcal{D}$ are defined as follows

$$
\mathcal{C}:=\mathfrak{R}^{n_{p}+n_{c}+n_{y}} \times\left[0, T_{2}\right], \quad \mathcal{D}:=\mathfrak{R}^{n_{p}+n_{c}+n_{y}} \times\{0\}
$$

The set-valued jump map allows to capture all possible transmission intervals of length within $T_{1}$ and $T_{2}$. Specifically, the hybrid model in (6) is able to characterize any sequence satisfying (2). Notice the definition of the jump map ensures that at each discrete event the output of the plant is stored into the variable $\hat{y}$.

At this stage, to simplify the analysis, we introduce the change of coordinates

$$
\eta:=y-\hat{y}
$$

which leads, by straightforward calculations, to the following closed-loop hybrid system in the new coordinates:

$$
\mathcal{H}_{c l} \begin{cases}\dot{x}=f(x) & x \in \mathcal{C} \\ x^{+} \in G(x) & x \in \mathcal{D}\end{cases}
$$

where $x:=\left(x_{c l}, \eta, \tau\right) \in \mathcal{X}:=\mathfrak{R}^{n_{p}+n_{c}+n_{y}+1}$ is the state, and $x_{c l}:=\left(x_{p}, x_{c}\right)$. The flow map is given by

$$
f(x):=\left(\mathbb{A} x_{c l}+\mathbb{B} \eta, \mathbb{J} x_{c l}+H \eta,-1\right) \quad \forall x \in \mathcal{C}
$$

where

$$
\begin{gathered}
\mathbb{A}:=\left[\begin{array}{c|c}
A_{p}+B_{p} D_{c} C_{p} & B_{p} C_{c} \\
\hline B_{c} C_{p} & A_{c}
\end{array}\right], \quad \mathbb{B}:=-\left[\begin{array}{c}
B_{p} D_{c} \\
\hline B_{c}
\end{array}\right] \\
\mathbb{J}:=\left[C_{p} A_{p}-H C_{p} \mid-E\right]
\end{gathered}
$$

derive from (1), (3), (4), and (7). The jump map is defined for all $x \in \mathcal{D}$ as $G(x):=\left(x_{c l}, 0,\left[T_{1}, T_{2}\right]\right)$. Observe that, as shown in Fig $2 \mathcal{H}_{c l}$ can be interpreted as the feedback interconnection of two dynamical systems, $\Sigma_{x_{c l}}$ and $\Sigma_{\eta}$. In particular, $\Sigma_{x_{c l}}$ is a continuous-time system described by:

$$
\Sigma_{x_{c l}}: \quad \dot{x}_{c l}=\mathbb{A} x_{c l}+\mathbb{B} \eta
$$

whereas $\Sigma_{\eta}$ is a hybrid dynamical system given as follows:

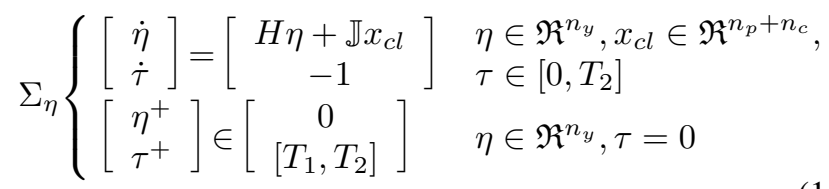

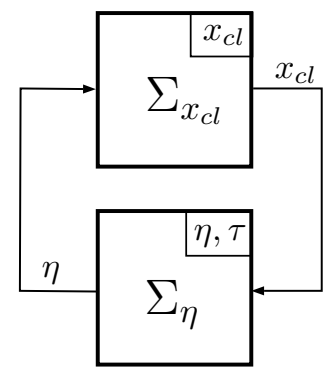

Fig. 2: Representation of $\mathcal{H}_{c l}$ as the interconnection of the dynamical systems $\Sigma_{x_{c l}}$ and $\Sigma_{\eta}$.

It is worth mentioning that considering $\mathcal{H}_{c l}$ as the interconnection of $\Sigma_{x_{c l}}$ and $\Sigma_{\eta}$ allows us to address stability analysis of the closed-loop system by employing an approach that is reminiscent of an "input-to-state stability small gain" philosophy. A conceptually similar approach can be found in [8].

\section{Problem Statement}

In this paper, we rely on the notion of global exponential stability $(G E S)$ of closed sets for a generic hybrid system $\mathcal{H}$ with state in $\mathfrak{R}^{n}$; see [12] for further details.

Definition 1 (Global exponential stability [12]). Let $\mathcal{A} \subset \mathfrak{R}^{n}$ be closed. The set $\mathcal{A}$ is globally exponentially stable (GES) for the hybrid system $\mathcal{H}$ if there exist strictly positive real numbers $\lambda$ and $k$ such that for any initial condition every maximal solution $\phi$ to $\mathcal{H}$ is complete and satisfies:

$$
|\phi(t, j)|_{\mathcal{A}} \leq k e^{-\lambda(t+j)}|\phi(0,0)|_{\mathcal{A}} \quad \forall(t, j) \in \operatorname{dom} \phi
$$

To design the holding device $\mathcal{J}$ and the controller $\mathcal{K}$, our approach aims at achieving that the following compact se 1

$$
\mathcal{A}:=\{0\} \times\{0\} \times\left[0, T_{2}\right]
$$

\footnotetext{
${ }^{1}$ Notice that, by definition of the system $\mathcal{H}_{c l}$ and of the set $\mathcal{A}$, for all $x \in \mathcal{C}$, one has $|x|_{\mathcal{A}}=\left|\left(x_{c l}, \eta\right)\right|$.
} 
is GES for the hybrid system $\mathcal{H}_{c l}$. In particular, the problem we solve is as follows:

Problem 1. Given the plant $\mathcal{P}$ in (1), design

$$
\Delta_{\mathcal{K}}:=\left[\begin{array}{c|c}
A_{c} & B_{c} \\
\hline C_{c} & D_{c}
\end{array}\right], \quad \Delta_{\mathcal{J}}=[H \mid E]
$$

such that the set $\mathcal{A}$ is GES for the hybrid system $\mathcal{H}_{c l}$ in (8) with the largest achievable value of $T_{2}$.

Observe that by definition, GES of $\mathcal{A}$ implies GES of the origin of the plant and controller systems.

\section{MAIN RESULTS}

\section{A. Lyapunov-based Stability Analysis}

Sufficient conditions to show exponential stability of the set $\mathcal{A}$ can already be derived by using results in [13]. However, those results would lead to matrix inequalities having the controller's and holding device's parameters appearing in a nonlinear fashion. Therefore, those conditions are, in general, not computationally tractable to provide a viable solution to Problem 1. With the purpose of obtaining more tractable conditions, in this paper, we consider the closedloop hybrid system $\mathcal{H}_{c l}$ as the interconnection depicted in Fig. 2. Moreover, we exploit such a structural characteristic of $\mathcal{H}_{c l}$ to introduce conditions on the individual systems $\Sigma_{x_{c l}}$ and $\Sigma_{\eta}$, and their interconnection such that the set $\mathcal{A}$ is $G E S$ for $\mathcal{H}_{c l}$. In particular, consider the following property:

Property 1. There exist continuously differentiable functions $W_{1}: \mathfrak{R}^{n_{p}+n_{c}} \rightarrow \mathfrak{R}$ and $W_{2}: \mathfrak{R}^{n_{y}+1} \rightarrow \mathfrak{R}$, positive definite functions $\rho_{1}: \mathfrak{R}^{n_{p}+n_{c}} \rightarrow \mathfrak{R}$ and $\omega_{1}: \mathfrak{R}^{n_{y}} \rightarrow \mathfrak{R}$, functions $\rho_{2}: \mathfrak{R}^{n_{y}} \rightarrow \mathfrak{R}$ and $\omega_{2}: \mathfrak{R}^{n_{c}+n_{p}} \rightarrow \mathfrak{R}$, and positive scalars $\bar{c}_{w_{1}}, \bar{c}_{w_{2}}, \underline{c}_{w_{1}}, \underline{c}_{w_{2}}, k_{w_{1}}$, and $k_{w_{2}}$ such that

$$
\begin{aligned}
& \underline{c}_{w_{1}}\left|x_{c l}\right|^{2} \leq W_{1}\left(x_{c l}\right) \leq \bar{c}_{w_{1}}\left|x_{c l}\right|^{2}, \quad \forall x_{c l} \in \mathfrak{R}^{n_{p}+n_{c}} \\
& \underline{c}_{w_{2}}|\eta|^{2} \leq W_{2}(\eta, \tau) \leq \bar{c}_{w_{2}}|\eta|^{2}, \quad \forall(\eta, \tau) \in \mathfrak{R}^{n_{y}} \times\left[0, T_{2}\right]
\end{aligned}
$$$$
\left\langle\nabla W_{1}\left(x_{c l}\right), \mathbb{A} x_{c l}+\mathbb{B} v_{1}\right\rangle \leq-\rho_{1}\left(x_{c l}\right)+\rho_{2}\left(v_{1}\right)
$$$$
\forall\left(x_{c l}, v_{1}\right) \in \mathfrak{R}^{n_{p}+n_{c}+n_{y}}
$$

$$
\begin{aligned}
\left\langle\nabla W_{2}(\eta, \tau),(H \eta+\right. & \left.\left.\mathbb{J} v_{2},-1\right)\right\rangle \leq-\omega_{1}(\eta)+\omega_{2}\left(v_{2}\right), \\
\forall\left(\eta, \tau, v_{2}\right) & \in \mathfrak{R}^{n_{y}} \times\left[0, T_{2}\right] \times \mathfrak{R}^{n_{p}+n_{c}}
\end{aligned}
$$$$
-\rho_{1}\left(x_{c l}\right)+\omega_{2}\left(x_{c l}\right) \leq-k_{w_{1}}\left|x_{c l}\right|^{2}, \quad \forall x_{c l} \in \mathfrak{R}^{n_{p}+n_{c}}
$$

$$
-\omega_{1}(\eta)+\rho_{2}(\eta) \leq-k_{w_{2}}|\eta|^{2}, \quad \forall \eta \in \mathfrak{R}^{n_{y}}
$$

Remark 1. It is worth mentioning that conditions in Property 11 resemble Lyapunov-like dissipation inequalities. In particular, conditions (16) and (17) resemble to input-output dissipation inequalities for, respectively, $\Sigma_{x_{c l}}$ and $\Sigma_{\eta}$. Relations (18) and (19) are reminiscent of small gain conditions on $\Sigma_{x_{c l}}$ and $\Sigma_{\eta}$, respectively.

\footnotetext{
${ }^{2}$ There is a typo in equation (16) in the conference proceeding, i.e., -1 is missing in the inner product.
}

Theorem 1. Let Property 1 hold. Then, the set $\mathcal{A}$ in (12) is GES for the hybrid closed-loop system $\mathcal{H}_{c l}$ in (8).

Sketch of the proof. For all $x \in \mathcal{X}$, define $V(x):=$ $W_{1}\left(x_{c l}\right)+W_{2}(\eta, \tau)$ and let

$$
\begin{aligned}
& \chi_{1}:=\min \left\{\underline{c}_{w_{1}}, \underline{c}_{w_{2}}\right\} \\
& \chi_{2}:=\max \left\{\bar{c}_{w_{1}}, \bar{c}_{w_{2}}\right\}
\end{aligned}
$$

Then, using (14) and (15) for all $x \in \mathcal{C}$, one gets

$$
\chi_{1}|x|_{\mathcal{A}}^{2} \leq V(x) \leq \chi_{2}|x|_{\mathcal{A}}^{2}
$$

Moreover, by using (15), for each $g=\left(x_{c l}, 0, w\right) \in G(x)$, $x=\left(x_{c l}, \eta, \tau\right) \in \mathcal{D}$ one has

$$
V(g)-V(x)=W_{2}(0, w)-W_{2}(\eta, 0) \leq-\bar{c}_{w_{2}}|\eta|^{2}
$$

Now observe that, from (16) and (17), for all $x \in \mathcal{C}$

$$
\langle\nabla V(x), f(x)\rangle \leq-k_{w_{1}}\left|x_{c l}\right|^{2}-k_{w_{2}}|\eta|^{2}
$$

which gives for all $x \in \mathcal{C}$

$$
\langle\nabla V(x), f(x)\rangle \leq-\underbrace{\min \left\{k_{w_{1}}, k_{w_{2}}\right\}}_{\chi_{3}}|x|_{\mathcal{A}}^{2}
$$

Combining (20), (21), and (22) and using (2) enable to conclude the proof.

With the purpose of deriving constructive design algorithms for the controller and the holding device, we perform a particular choice for the functions $W_{1}$ and $W_{2}$ in Property 1 In particular, let $P_{1} \in \mathrm{S}_{+}^{n_{p}+n_{c}}, P_{2} \in \mathrm{S}_{+}^{n_{y}}$, and $\delta$ a positive real number. Inspired by [10], we make the following selection:

$$
W_{1}\left(x_{c l}\right):=x_{c l}^{\top} P_{1} x_{c l}, \quad W_{2}(\eta, \tau):=e^{\delta \tau} \eta^{\top} P_{2} \eta
$$

The structure of the selected functions $W_{1}$ and $W_{2}$ allows to provide sufficient conditions for exponential stability of the set $\mathcal{A}$ in the form of matrix inequalities. To this end, consider the following proposition:

Proposition 1. If there exist $P_{1}, S, R \in \mathrm{S}_{+}^{n_{p}+n_{c}}, P_{2}, Q, T \in$ $\mathrm{S}_{+}^{n_{y}}$, a positive real number $\delta$, and matrices $A_{c} \in \mathfrak{R}^{n_{c} \times n_{c}}$, $B_{c} \in \mathfrak{R}^{n_{c} \times n_{y}}, C_{c} \in \mathfrak{R}^{n_{u} \times n_{c}}, D_{c} \in \mathfrak{R}^{n_{u} \times n_{y}}, H \in \mathfrak{R}^{n_{y} \times n_{y}}$, and $E \in \mathfrak{R}^{n_{y} \times n_{c}}$, such that

$$
\begin{gathered}
Q-T \prec 0 \\
R-S \prec 0 \\
\mathcal{M}_{1}:=\left[\begin{array}{cc}
\operatorname{He}\left(P_{1} \mathbb{A}\right)+S & P_{1} \mathbb{B} \\
\bullet & -Q
\end{array}\right] \preceq 0 \\
\mathcal{M}_{2}(0) \preceq 0, \quad \mathcal{M}_{2}\left(T_{2}\right) \preceq 0
\end{gathered}
$$

where

$$
\left[0, T_{2}\right] \ni \tau \mapsto \mathcal{M}_{2}(\tau):=\left[\begin{array}{cc}
\left(\mathrm{He}\left(P_{2} H\right)-\delta P_{2}\right) e^{\delta \tau}+T & P_{2} \rrbracket e^{\delta \tau} \\
\bullet & -R
\end{array}\right]
$$

then Property 1$]$ holds. 


\section{B. LMI-based Controller Design}

In the previous section, sufficient conditions were provided to guarantee exponential stability of $\mathcal{A}$ for $\mathcal{H}_{c l}$. In particular, through Proposition 1 , these conditions turn into the feasibility problem of matrix inequalities, which are not suitable to be a tool for the solution of Problem 1 because they are nonlinear in the variables $P_{1}, P_{2}, A_{c}, B_{c}, C_{c}, D_{c}, H, E, \delta$. Therefore, further manipulations are needed to derive a computationally efficient design procedure for the controller. While the nonlinearity in $\delta$ can be easily overcome through a line search, other nonlinearities must be properly treated. To this end, in the following, we provide sufficient conditions to turn the solution to Problem 1 into the feasibility problem of some LMIs.

Lemma 1. Let $F \in \mathrm{S}_{+}^{n}$. Then, for any $\alpha \in \mathfrak{R}$ the following relation holds:

$$
F^{-1}-2 \alpha \mathbf{I}+\alpha^{2} F \succeq 0
$$

Theorem 2. Given the plant (1), and scalars $\delta>0$ and $\alpha$, suppose there exist $P_{2}, T, Q \in \mathrm{S}_{+}^{n_{y}}, R, F \in \mathrm{S}_{+}^{2 n_{p}}, X, Y \in$ $\mathrm{S}_{+}^{n_{p}}, K \in \mathfrak{R}^{n_{p} \times n_{p}}, L \in \mathfrak{R}^{n_{p} \times n_{y}}, M \in \mathfrak{R}^{n_{u} \times n_{p}}, N \in$ $\mathfrak{R}^{n_{u} \times n_{y}}, J \in \mathfrak{R}^{n_{y} \times n_{y}}, Z \in \mathfrak{R}^{n_{y} \times n_{p}}$, and a nonsingular matrix $V \in \mathfrak{R}^{n_{p} \times n_{p}}$, such that

$$
\begin{aligned}
& \Theta:=\left[\begin{array}{ll}
Y & \mathbf{I} \\
\mathbf{I} & X
\end{array}\right] \succ 0 \\
& Q-T \prec 0 \\
& R-2 \alpha I+\alpha^{2} F \prec 0 \\
& \widehat{\mathcal{M}}_{1}:=\left[\begin{array}{ccc}
\operatorname{He}(\Lambda) & \Pi & \Phi^{\top} \\
\bullet & -Q & 0 \\
\bullet & \bullet & -F
\end{array}\right] \preceq 0 \\
& \widehat{\mathcal{M}}_{2}(0) \preceq 0, \quad \widehat{\mathcal{M}}_{2}\left(T_{2}\right) \preceq 0
\end{aligned}
$$

where

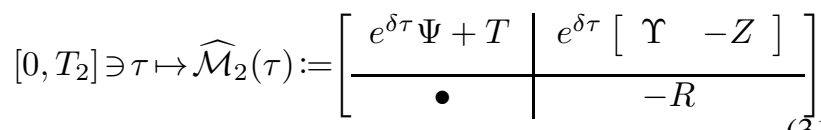

$$
\begin{aligned}
& \Phi:=\left[\begin{array}{cc}
Y & I \\
V^{\top} & 0
\end{array}\right] \\
& \Lambda:=\left[\begin{array}{cc}
A_{p} Y+B_{p} M & A_{p}+B_{p} N C_{p} \\
K & X A_{p}+L C_{p}
\end{array}\right], \quad \Pi:=-\left[\begin{array}{c}
B_{p} N \\
L
\end{array}\right] \\
& \Psi:=\operatorname{He}(J)-\delta P_{2}, \quad \Upsilon:=P_{2} C_{p} A_{p}-J C_{p}
\end{aligned}
$$

Then, the matrix $\mathbf{I}-X Y$ is nonsingular. Let $U \in \mathfrak{R}^{n_{p} \times n_{p}}$ be any nonsingular matrix such that

$$
X Y+U V^{\top}=\mathbf{I}
$$

Then, the conditions in Proposition 1$]$ are satisfied; hence, Property 1 holds. Furthermore, selecting $\Delta_{\mathcal{K}}$ and $\Delta_{\mathcal{J}}$ as in (35) (at the top of the page) solves Problem 1
Remark 2. Theorem 2 requires matrix $V$ to be nonsingular. Although this constraint is hard to formulate in an LMI setting, nonsingularity of $V$ can be easily enforced, e.g., by considering the following constraint $V+V^{\top} \succ 0$.

Notice that when $\delta, \alpha$, and $T_{2}$ are fixed, the conditions in Theorem 2 become LMIs. As such, when one is interested in enlarging $T_{2}$, Theorem 2 can be employed to design the controller gains to solve the following optimization problem:

$$
\begin{array}{cc}
\underset{\delta, \alpha, P_{2}, T, Q, R, F, X, Y, K, L, M, N, J, Z, V}{\operatorname{maximize}} & T_{2} \\
\text { subject to } &
\end{array}
$$

The above optimization problem can be efficiently solved via semidefinite programming, with the only caveat of performing a line search on scalar parameters $\delta, \alpha$, and $T_{2}$.

\section{NuMERICAL EXAMPLE}

In this section, we showcase the proposed design approach for a double integrator plant, i.e.

$$
\left[\begin{array}{l|l|l}
A_{p} & B_{p} & C_{p}^{\top}
\end{array}\right]=\left[\begin{array}{ll|l|l}
0 & 1 & 0 & 1 \\
0 & 0 & 1 & 0
\end{array}\right]
$$

Numerical solutions to LMIs are obtained through the solver SDPT3 [14] and coded in Matlab via YALMIP [15]. Simulations of hybrid systems are performed in Matlab via the Hybrid Equations (HyEQ) Toolbox [16].

With the objective of avoiding ill conditioned controller parameters, we consider the following additional constraint:

$$
-50 \Theta \preceq \operatorname{He}(\Lambda) \preceq-\Theta
$$

where $\Lambda$ is defined in (33). In particular, the above constraint ensures that the $\operatorname{spec}(\mathbb{A})$, which characterizes the closed-loop continuous-time dynamics, is contained in the set $\{s \in \mathbb{C}$ : $-25 \leq \mathfrak{R e}(s) \leq-0.5\}$; see [9].

By pursing this approach, our design methodology leads to: $T_{2}=0.6, \delta=3, \alpha=0.5263$, and the following values of the controller parameters:

$$
\begin{aligned}
& \Delta_{\mathcal{K}}=\left[\begin{array}{cc|c}
-51.8741 & 2.3076 & 1.2822 \\
-435.5136 & 4.8396 & 10.5892 \\
\hline 826.2669-90.2077 & -21.4983
\end{array}\right] \\
& \Delta_{\mathcal{J}}=\left[\begin{array}{lll}
-0.4553 \mid-0.0041 & 0.0008
\end{array}\right]
\end{aligned}
$$

To visualize the behavior of the closed-loop system with the controller in 37, in Fig. 3 we report the evolution of the plant state $x_{p}$, the state of the holding device $\hat{y}$, the control signal $u$, and the timer variable $\tau$. In this simulation, $x_{p}(0,0)=(0.85,0.52), x_{c}(0,0)=0, \hat{y}(0,0)=0, T_{1}=$ 0.01 , and transmission intervals are selected between $T_{1}$ and $T_{2}$ accordingly to a sinusoidal law with frequency 10.5 . Fig. (4) depicts the evolution of the Lyapunov function $V$ used in the proof Theorem 11 along the same solution considered here above. As expected, $V$ is nonincreasing and exponentially converges to zero. 


$$
\Delta_{\mathcal{K}}=\left[\begin{array}{cc|c}
U^{-1} & -U^{-1} X B_{p} \\
0 & \mathbf{I}
\end{array}\right]\left[\begin{array}{cc}
K-X A_{p} Y & L \\
\hline M & N
\end{array}\right]\left[\begin{array}{cc}
V^{-\top} & 0 \\
-C_{p} Y V^{-\top} & \mathbf{I}
\end{array}\right], \quad \Delta_{\mathcal{J}}=\left[P_{2}^{-1} J \mid P_{2}^{-1} Z\right]
$$

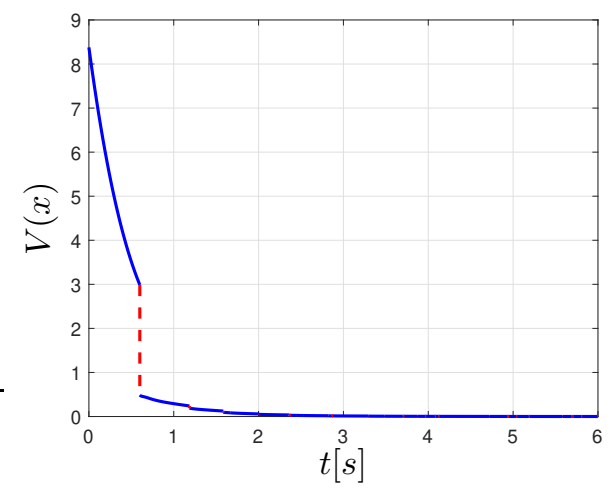

Fig. 4: Evolution of the Lyapunov function used in the proof of Theorem 1
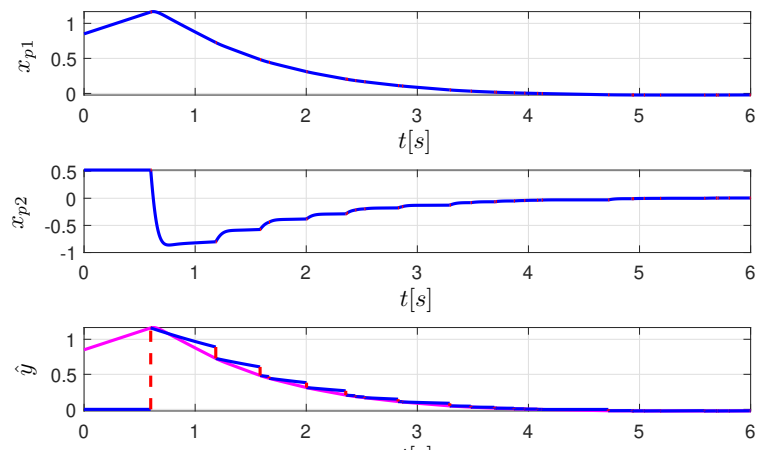

$t[s]$
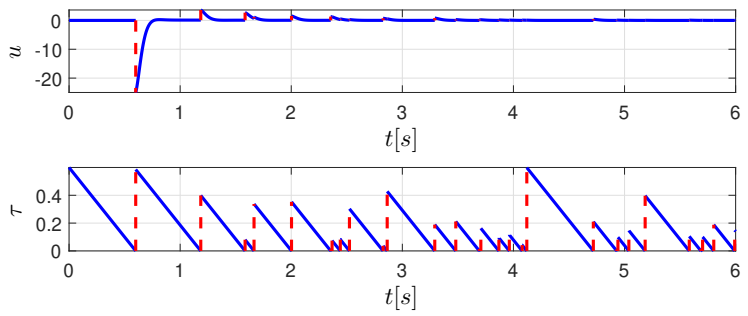

Fig. 3: Response of the closed-loop system. From the top to the bottom: plant state $x_{p}$, state of the holding device $\hat{y}$ (blue) and plant output $y$ (magenta), control signal $u$, and timer variable $\tau$.

\section{CONCLUSION}

In this paper, we investigated the problem of designing output feedback controllers for linear time-invariant systems where measurements are available in an aperiodic intermittent fashion. In particular, our design methodology aims at obtaining a controller such that the closed-loop NCS has a set globally exponentially stable with the largest achievable interval without measurements. These results are accomplished by relying on Lyapunov results for hybrid dynamical systems. Moreover, by employing an approach that is reminiscent of an "input-to-state stability small gain" philosophy, we obtained sufficient conditions that are suitably converted into LMIs. The effectiveness of the proposed approach is showcased throughout a numerical example.

\section{REFERENCES}

[1] J. P. Hespanha, P. Naghshtabrizi, and Y. Xu, "A survey of recent results in networked control systems," Proceedings of the IEEE, vol. 95, no. 1, pp. 138-162, 2007.

[2] L. Hetel, C. Fiter, H. Omran, A. Seuret, E. Fridman, J.-P. Richard, and S. I. Niculescu, "Recent developments on the stability of systems with aperiodic sampling: An overview," Automatica, vol. 76, pp. 309-335, 2017.

[3] D. B. Dačić and D. Nešić, "Quadratic stabilization of linear networked control systems via simultaneous protocol and controller design," Automatica, vol. 43, no. 7, pp. 1145-1155, 2007

[4] M. Donkers, J. Daafouz, and W. Heemels, "Output-based controller synthesis for networked control systems with periodic protocols and time-varying transmission intervals and delays," IFAC Proceedings Volumes, vol. 47, no. 3, pp. 6478-6483, 2014.

[5] E. Fridman, U. Shaked, and V. Suplin, "Input/output delay approach to robust sampled-data $\mathcal{H}_{\infty}$ control," Systems \& Control Letters, vol. 54, no. 3, pp. 271-282, 2005.

[6] F. Ferrante, F. Gouaisbaut, R. G. Sanfelice, and S. Tarbouriech, "Observer-based control design for linear systems in the presence of limited measurement streams and intermittent input access," in Proceedings of the American Control Conference, 2015, pp. 46894694.

[7] C. Briat, "Spectral necessary and sufficient conditions for samplingperiod-independent stabilisation of periodic and aperiodic sampleddata systems using a class of generalised sampled-data hold functions," International Journal of Control, vol. 87, no. 3, pp. 612-621, 2014.

[8] D. Carnevale, A. Teel, and D. Nešić, "A Lyapunov proof of an improved maximum allowable transfer interval for networked control systems," IEEE Transactions on Automatic Control, vol. 52, no. 5, pp. 892-897, 2007.

[9] C. Scherer, P. Gahinet, and M. Chilali, "Multiobjective outputfeedback control via LMI optimization," IEEE Transactions on Automatic Control, vol. 42, no. 7, pp. 896-911, 1997.

[10] F. Ferrante, F. Gouaisbaut, R. G. Sanfelice, and S. Tarbouriech, " $\mathcal{L}_{2}$ state estimation with guaranteed convergence speed in the presence of sporadic measurements," IEEE Transactions on Automatic Control, vol. 64, no. 8, pp. 3362-3369, 2019.

[11] R. Goebel, R. G. Sanfelice, and A. R. Teel, Hybrid Dynamical Systems: Modeling, Stability, and Robustness. Princeton University Press, 2012.

[12] A. R. Teel, F. Forni, and L. Zaccarian, "Lyapunov-based sufficient conditions for exponential stability in hybrid systems," IEEE Transactions on Automatic Control, vol. 58, no. 6, pp. 1591-1596, 2012.

[13] R. Merco, F. Ferrante, and P. Pisu, "On DoS resiliency analysis of networked control systems: Trade-off between jamming actions and network delays," IEEE Control Systems Letters, vol. 3, no. 3, pp. 559564, July 2019

[14] R. H. Tütüncü, K.-C. Toh, and M. J. Todd, "Solving semidefinitequadratic-linear programs using SDPT3," Mathematical Programming, vol. 95, no. 2, pp. 189-217, 2003.

[15] J. Lofberg, "Yalmip: A toolbox for modeling and optimization in Matlab," in International Symposium on Computer Aided Control Systems Design, 2004, pp. 284-289.

[16] R. G. Sanfelice, D. Copp, and P. Nanez, "A toolbox for simulation of hybrid systems in Matlab/Simulink: Hybrid equations (hyeq) toolbox," in Proceedings of the 16th International Conference on Hybrid systems: Computation and Control. ACM, 2013, pp. 101-106. 